\section{LETTER TO THE EDITOR: RESPONSE TO "SURGEON UNEMPLOYMENT: WOULD PRACTICE SHARING BE A VIABLE SOLUTION?"}

We read with great interest the article by Wakeam and Feinberg ${ }^{1}$ regarding a shared practice model whereby a surgeon who is winding down practice partners with a young surgeon.

Does this type of practice model need only apply to a transition into and out of practice? Are there other forms of sharing a practice where efficient use of resources and mentoring can occur? Our shared practice model may represent an alternative.

Although our clinic, endoscopy and operative resources have not increased proportionally as we hire surgeons to our colorectal group, complete clinical integration has afforded us a work environment that is efficient, fun and, most importantly, provides great patient care. There is a 1-line system to "The Ottawa Colorectal Group," whereby 1 surgeon in our group may provide the initial consultation and obtain consent for an operative procedure, which may be performed by any of the 3 surgeons (soon to be 4); our offices and administrative support are all in 1 area. At times, all 3 of us are in clinic together, but invariably there are extra operative days, endoscopy time, academic activities or vacation that usually lead to 2 surgeons working together in clinic. Consequently, a surgeon in our group may meet a patient for the first time on the day of an operation and therefore must rely on a partner to establish a therapeutic relationship. With an automatic second opinion available at the time of consult, or when we run the list of patients with their management plans (at the end of clinic with the entire team, including our administrative staff), patients are happy to know that multiple surgeons are involved in their care. This also extends to the operating room, where we are consistently in each other's rooms. There is also "round the clock" coverage of patients in all clinical settings (i.e., clinic to operating room), as the schedule is set up to ensure that at least 1 surgeon is always available. Hence, resources are not wasted, and inpatients receive consistent care through a rotating weekly coverage schedule (which is really nice from a lifestyle perspective). As eluded to, we are able to capture extra resources that become available, and now, as we expand to a fourth surgeon, we are extending beyond the academic centre to work with a large community hospital where, as a group, we will have privileges and regular operative time. This new partnership will not only foster mentorship within our group but also provide a means to mentor community-based surgeons wishing to learn new techniques in colorectal surgery, thereby enhancing patient care throughout our region. The right people are crucial for this model to work, as it is built on trust - personalities, technical skills, judgment and core value systems need to be aligned. We also need to communicate regularly and frequently a good electronic medical record would facilitate improvement.

As general surgeons, we see this concept in action on the acute care service, which is replacing the traditional emergency surgery model; our shared practice represents a similar evolution within the elective setting.

\section{Husein Moloo, MD; Isabelle Raiche, MD; Lara Williams, MD; Fady Balaa, MD}

From the Department of Surgery, the Ottawa Hospital, Ottawa, Ont.

DOI: $10.1503 /$ cjs.006216

\section{References}

1. Wakeam E, Feinberg S. Surgeon unemployment: Would practice sharing be a viable solution? Can $\mathcal{F}$ Surg 2016;59:141-2.

\section{AUthOR RESPONSE}

Dr. Moloo and colleagues of the Ottawa Colorectal Group have posed an interesting question about how we can redesign practice models to maximize mentoring.
Shared practices need not be restricted to surgeons coming into and out of practice. They describe another method of shared practice that would be useful in ensuring better mentoring and quality patient care. General surgery is an intense medical specialty where the maxim of "one sick patient is the difference between being not busy enough and too busy" is lived every day. As we evolve, we need to look to different ways of practising. We suggested a "job-share" as a way of integrating new graduates into practice with senior mentors, and Dr. Mooloo's group has taken this shared practice to the next level.

One important consideration, however, is that the model we propose is a response to a lack of permanent employment positions. Of course, the benefits of additional mentorship and more flexible work hours in the early stages of practice to accommodate family life are perks of this system that apply regardless of the job situation; the most ideal circumstance is one in which all new graduates would have permanent positions that meet their needs and those of their patients. If we understand the model being proposed by Dr. Moloo and colleagues, it will not decrease the employment pressures on new graduates, though it may have other benefits.

Whether this works for all surgeons and patients will depend on philosophy of care and culture (e.g., in our opinion it is not ideal to meet a patient for the first time at the doors to the operating room, but this is purely a matter of preference). For the Ottawa model to work, it requires excellent communication, patient education and a consistent approach to care.

\section{Elliot Wakeam, MD, MPH; Stan Feinberg, MD}

From the General Surgery Residency Program, University of Toronto, Toronto, Ont. (Wakeam); and the Division of General Surgery, North York General Hospital, University of Toronto, Toronto, Ont. (Feinberg).

DOI: $10.1503 /$ cjs.009416 